\title{
The old question: which programming language should we choose to teach to program?
}

\author{
Sónia Rolland Sobral [0000-0002-5041-3597] \\ ${ }^{1}$ REMIT, Universidade Portucalense, Porto, Portugal \\ sonia@upt.pt
}

\begin{abstract}
When students enter higher education in computer courses, students have, in the first semester, to perceive and streamline computer (or computational) thinking the names for the unit are diverse, usually algorithm and programming, introduction to programming or even Programming I or algorithm. In research we call CS1, or computer science 1 , since that designation was used in an important document with curriculum recommendations prepared by the Association for Computing Machinery (ACM) in 1978. Despite different names, it is intended with these units that students become start in the world of programming However, if the learning objectives do not differ much, the same cannot be said of the adapted programming language. There is not, nor is it likely to be, a consensus regarding the programming language used by the student's startup in the programming world. The various documents with curriculum recommendations for computer science from ACM and the Institute of Electrical and Electronic Engineers (IEEE) do not clearly define which programming language to adopt. Those responsible for the courses must make this choice, consciously and not just following industry trends. The purpose of this article is to draw a picture of the adoption of the different programming languages that have been used over time, as well as to help in choosing the most accurate and conscious option possible.
\end{abstract}

Keywords: Programming Languages, Undergraduate Studies; Introduction to Programming.

Sobral, Sónia Rolland (2021). The old question: which programming language should we choose to teach to program? ICADS 2021 - The 2021 International Conference on Advances in Digital Science (https://ics.events/icads-2021/) 